Overrun réel total :

Overrun réel \% :

$$
255-216,160=38,840 \text {. }
$$

$$
\frac{38,840 \times 100}{216,160}=17,96 \% \text {. }
$$

[16] Différence entre [14] et [15].

Cette différence joue également un rôle important dans l'étude du rendement, aussi doit-elle figurer sur le livre afin d'être comparée avec celle des jours précédents.

$$
19,68-17,96=1,72 .
$$

Ces calculs, qui, à première vue, paraîssent un peu compliqués, sont au contraire, comme nous venons de le voir, extrêmement simples pour le Directeur de laiterie, surtout lorsqu'il a fait prendre à son personnel la bonne habitude d'exécuter régulièrement et intelligemment les jaugeages, prélèvements et analyses et de les lui fournir en totalité tous les jours. Nous pouvons affirmer par expérience que, lorsqu'on est en possession de ces renseignements, il ne faut pas plus de 12 minutes pour établir complètement le rendement d'une journée.

Ces calculs permettent de fonder une appréciation très sûre sur la marche de l'usine, ils éveillent la sagacité de Directeur, maintiennent le parsonnel vigilant, donnent des indices précieux sur les fautes et les négligences commises ou sur les difficultés rencontrées aux différents postes.

(A suivre.)

\title{
LE DOSAGE DE L'IODE DANS LE LAIT
}

parle Dr Th. von FELLENBERG,

du Laboratoire de Chimie du Service fédéral de l'Hygiène publique à Berne

Le dosage de minimes quantités d'iode dans les produits organiques s'effectue en détruisant la matière organique par combustion en présence d'un alcali, extraction par l'alcool de l'iodure formé, puis dosage titrimétrique ou colorimétrique de l'iode.

Tous les réactifs employés doivent être préalablement purifiés de façon à ne pas contenir d'iode. L'alcool et l'eau seront distillés en présence d'un peu de carbonate de potassium. Le carbonate de potassium en solution concentrée sera agité à 6 reprises a vec son volume d'alcool à $95^{\circ}$, dans un entonnoir à robinet. Il est très difficile de purifier la potasse caustique. Le mieux est de choisir parmi les potasses celle qui renferme le moins d'iode et de tenir compte de sa teneur en jode dans les calculs. L'essai des potasses caustiques se fait en les transformant en carbonate de potassium et extraction de la solution concentrée par l'alcool. 
Avec le lait, vu sa teneur en matière grasse, il faut procéder à une saponification préalable par la potasse caustique. Cette saponification peut s'effectuer à froid.

Dans un petit ballon, on verse $25 \mathrm{~cm}$. de lait et $2,5 \mathrm{~cm}$. de solution saturée de potasse caustique, on abandonne le tout pendant quelques jours et l'on agite 2 à 3 fois par jour, puis on chauffe au bain-marie en adaptant sur le ballon un réfrigérant en forme de poire, consistant en un tube de verre, ouvert à l'une de ses extrémités, effilé légèrement à. sa partie inférieure et évasé en forme de poire dans sa partie médiane.

Il peut s'adapter à n'importe quelle forme de ballon.

Le lait saponifié est évaporé avec précaution au feu nu d'un petit bec dans une capsule de fer ou de nickel de 8 à $10 \mathrm{~cm}$. de diamètre. Lorsque l'eau est éva porée, on élève la température jusqu'à ce que la masse soit transformée en charbon. On évitera que la matière s'enflamme, si cela était on recouvrirait la capsule d'une plaque de fer.

On écrase alors le charbon (dans la capsule, avec un pilon de verre), puis on continue à chauffer, et, en remuant au besoin avec une baguette de verre, on veillera à ce que toutes les particules soient chauffées également. Lorsqu'il ne se dégage plus de vapeurs on retire la capsule du feu, la recouvre avec une capsule de plus fort diamètre et la porte dans la partie demi-sombre d'un four à moufle chauffé de la façon suivante : le four est porté au rouge incandescent faible, puis on règle les flammes de façon qu'après quelques minutes on n'aperçoive plus que quelques points en faible incandescence. On laisse la capsule pendant 10-15 minutes dans le moufle puis on extrait le charbon, à plusieurs reprises, avec un peu d'eau en aspirant à la trompe. Le filtrat doit être incolore ou, tout au plus, jaune clair. On l'évapore à feu nu dans un becher en verre résistant de Ièna. Pendant ce temps, on porte le charbon extrait dans une nacelle en fer ou en nickel que l'on introduit dans un tube de quartz et l'on brûle dans un courant d'air bien réglé ; on utilise comme prolongement un tube à 10 boules contenant 0,25 gr. de solution saturée de carbonate de potassium et $40 \mathrm{~cm}^{3}$ d'eau.

Le tube que j'utilise a $40 \mathrm{~cm}$. de longueur et $2,5 \mathrm{~cm}$. de diamètre. Il est terminé par un coude à angle droit, long de $20 \mathrm{~cm}$. ayant un diamètre intérieur de $0,5 \mathrm{~cm}$. Derrière la nacelle contenant le charbon se trouve une feuille de platine enroulée en spirale, portée à rouge incandescent, ce qui favorise au maximum la combustion des matières volatiles. Le liquide contenu dans les boules doit être, en principe, complètement incolore.

Lorsque la combustion est terminée on retire la nacelle et les cendres du tube de quartz. On extrait les cendres à l'eau. Cet extrait, le liquide du tube à boules et l'eau nécessaire pour le lavage du tube de quartz, sont réunis dans le becher qui contenait le filtrat du charbon 
et évaporés à sec. On porte le becher pendant 5 minutes dans le moufle chauffé avec les précautions indiquées plus haut. On le retire avec une pince à creuset, préalablement chauffée de manière à ce qu'elle brunisse le bois, puis laisse refroidir sur une plaque d'amiante, ce qui demande 5 minutes environ. On dissout le résidu du becher dans très peu d'eau et évapore jusqu'à consistance pâteuse. On extrait à 4-5 reprises avec un peu d'alcool à $95 \%$, en agitant avec une baguette de verre. L'extrait alcoolique est évaporé en plusieurs portions dans une fiole d'Erlenmeyer de $50 \mathrm{~cm}^{3}$ en verre de Iéna. Le résidu est additionné de 1-2 gouttes de solution saturée de carbonate de potassium et reporté pendant 5 minutes dans le moufle. Pour la dernière fois, on extrait le résidu en l'humectant d'abord avec $0,5 \mathrm{~cm}$. environ d'alcool à $85 \%$, puis en l'épuisant à plusieurs reprises avec de l'alcool à $95 \%$. La solution alcoolique est évaporée dans une fiole de $50 \mathrm{~cm}$., puis chauffée une dernière fois au moufle.

On peut alors doser l'iode colorimétriquement ou titrimétriquement ou faire suivre le dosage colorimétrique d'un dosage titrimétrique.

- Pour le dosage colorimétrique on dissout le résidu dans la fioleErlenmeyer dans $0,3 \mathrm{~cm}$. d'eau et verse le liquide dans un tube dont l'extrémité est coupée en biseau, long de $80 \mathrm{~mm}$. et d'un diamètre interne de $5 \mathrm{~mm}$. On aura toujours en réserve une vingtaine de tubes, tous de même dimension. Il reste naturellement un peu de solution dans la fiole. On déterminera une fois pour toutes, par pesée, la quantité de solution restant et corrigera le résultat final en conséquence. A la solution dans le tube, on ajoute $0,02 \mathrm{~cm}$. de chloroforme au moyen d'une pipette de $0,1 \mathrm{~cm}^{3}$ divisée en $1 / 1000 \mathrm{~cm}^{3}$, puis une goutte de mélange nitreux et agite fortement, 80 fois environ. D'une main on tient le tube par une de ses extrémités tandis qu'avec $l$ autre main on frappe l'autre extrémité, le tube revient ainsi toujours à sa position primitive.

On prépare le mélange nitreux en dissolvant 1 à 2 cgr. de nitrite de sodium dans $10 \mathrm{~cm}^{3}$ d'acide sulfuriq ue $3 \times \mathrm{N}$.

Après agitation on centrifuge le tube et compare la coloration rouge-violet du chloroforme à celle de solutions de comparaison traitées de la même façon. Comme solution "standard " on utilise.une solution contenant $13,07 \mathrm{mgr}$. d'iodure de potassium (= $10 \mathrm{mgr}$. I) dans $100 \mathrm{~cm}^{3} ; 0,01 \mathrm{~cm}^{3}$ de cette solution correspond à $1 \gamma 1$ ( $1 \gamma=0,001$ mgr.).

On procède ensuite au dosage volumétriq ue. On ajoute à la solution dans le tube quelques gouttes d'eau bromée saturée pour faire passer l'iode à l'état d'acide iodique, puis verse le liquide dans une fiole d'Frlenmeyer, rince deux fois le tube avec un peu d'eau et dilue jusqu'à environ $5 \mathrm{~cm}^{3}$. On ajoute quelques fragments de pierre ponce 
et fait bouillir pour chasser l'excès de brome jusq u'à ce que le volume soit réduit à $1-1,5 \mathrm{~cm}$. On refroidit, ajoute quelq ues milligrammes d'iodure de potassium, puis une goutte d'une solution frâ̂shement préparée d'amidon soluble et titre en utilisant uue pipatte identique à celle employée pour mesurer le chloroforme et une solution $\mathrm{N} / 250$ d'hyposulfite de sodium. Comme chaque molécule d'acide iodique libère 6 atomes d'iode, on obtiendra done 6 fois la quantité d'iode présente.

Le titre de la solution d'hyposulfite est déterminé chaque fois en faisant un essai à blanc avec une quantité connue d'iode, à peu près égale à celle à déterminer.

\title{
SUR LES FLORES BACTÉRIENNE ET FONGIQUE DES CAILLÉS DE LAIT
}

\author{
RÔLE DES SYMBIOSES DANS LA MATURATION \\ DU CAMEMBERT \\ par \\ Mademoiselle FRANÇOISE SANSONETTI \\ Docteur en Pharmacie
}

(Suite)

\section{TROISIEME PARTIE}

\section{EFFETS DE L'ACTIVITÉ DES ORGANISMES SUR LE MILIEU}

Cette Partie est réservée à l'étude des transformations du milieu par le Penicillium, l'Ö̈dium, les ferments lactiques et leurs symbioses. Nous examinerons d'abord l'action des organismes sur la réaction du milieu et sur les sueres. Nous étudierons ensuite l'effet des diastases, et quelques as pects de l'attaque de la caséine.

\section{1. - ACtION SUR LA REACTION DU MILIEU}

A du sérum d'emprésurage, nous avons ajouté des indicateurs : $15 \%$ de teinture de tournesol, ou des colorants de l'échelle des $p H$ de ClaRK et LuBs, en qualité indiquée par les auteurs.

La zone de virage des indicateurs employés s'étendait depuis $p \mathrm{H}=3,0$ aे $p \mathrm{H}=9,6$. Ces colorants n'avaient aucun effet toxique sur les cultures ; mais celles-ci, modifiant le rouge de méthyle, toute variation comprise entre $p H=4,4$ et $p H=6$ passait ina perçue. Aussi dans une troisième expérience les indicateurs ont été supprimés. Le $p \mathrm{H}$ des cultures a été pris régulièrement suivant la technique habituelle de Clark et Lubs.

Conditions des cuttures. - Les milieux stérilisés à $115^{\circ}$ pendant $1 / 4$ d'heure sont ensemencés et portés à l'étuve à $24^{\circ}$. 